\title{
Vascular Glycocalyx Sodium Store - Determinant of Salt Sensitivity?
}

\author{
Hans Oberleithner Marianne Wilhelmi \\ Institute of Physiology II, Medical Faculty, University Münster, Münster, Germany
}

\section{Key Words}

Heparan sulfate - Hypertension - Salt blood test .

Erythrocyte shedding - Endothelial dysfunction

\begin{abstract}
Smart mechanisms allow frictionless slipping of rather rigid erythrocytes (red blood cells, RBC) through narrow blood vessels. Nature solved this problem in an elegant way coating the moving object (RBC) and the tunnel wall (endothelium) by negative charges (glycocalyx). As long as these surfaces are intact, repulsive forces create a 'security zone' that keeps the respective surfaces separated from each other. However, damage of either one of these surfaces causes loss of negative charges, allowing an unfavorable physical interaction between the RBC and the endothelium. It has been recently shown that any alteration of the endothelial glycocalyx leaves nasty footprints on the RBC glycocalyx. In this scenario, sodium ions hold a prominent role. Plasma sodium is stored in the glycocalyx partially neutralizing the negative surface charges. A 'good' glycocalyx has a high sodium store capacity but still maintains sufficient surface negativity at normal plasma sodium. A 'bad' glycocalyx shows the opposite. This concept was used for the development of the socalled 'salt blood test' (SBT) that quantitatively measures RBC sodium store capacity of the glycocalyx and thus indirectly evaluates the quality of the inner vessel wall. In an initial step, the applicability of the SBT was tested in eight dif-
\end{abstract}

ferent medical facilities. The study shows that an increased salt sensitivity, as measured by the SBT, is more frequently found in individuals with a hypertensive history, despite antihypertensive medication. Taken together, preservation of the endothelial glycocalyx appears to be of utmost importance for maintaining a well-balanced function of the vascular system.

(c) 2015 S. Karger AG, Basel

In the broadest sense, salt (i.e., sodium) sensitivity in man could be defined as the response of the cardiovascular system to sodium chloride intake $[1,2]$. A more precise definition is difficult since time course (hours to weeks), quality of response (e.g., change in blood pressure), and amounts of salt intake may vary. Most studies favor sodium ions $\left(\mathrm{Na}^{+}\right)$to be responsible for any saltinduced alterations of the vascular system [3]. High salt sensitivity, indicated by a significant increase of blood pressure in response to a salt load is estimated to occur in about $30-40 \%$ of the world's population [2]. Although under discussion for many decades, high salt sensitivity, as being a significant risk factor for the development of vascular-based diseases, is getting particular attention over the past years. This 'wake-up' call is possibly due to reports in the scientific literature that sodium 'per se' may act on different organs and tissues including kidney, heart, skin, lymphatics, and the vascular system [4-8].

\section{KARGER 125}

(C) 2015 S. Karger AG, Basel

$0253-5068 / 15 / 0393-0007 \$ 39.50 / 0$ 
Fig. 1. Schematic illustrating the interaction of red blood cells (RBC) with the inner wall of blood vessels (endothelium).

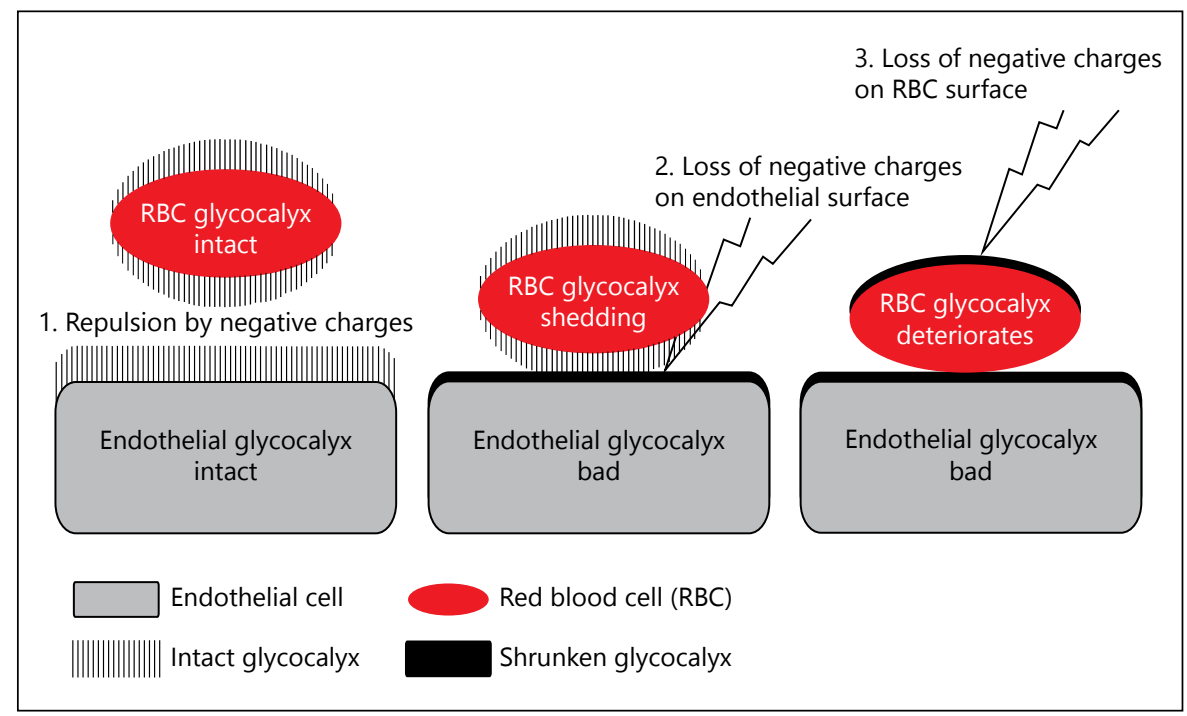

Vascular endothelium is sensitive to small changes in the plasma sodium concentration. The 'sodium sensor' is supposed to be located in the endothelium, probably closely associated with the occurrence of endothelial sodium channels $[9,11]$ and the negatively charged endothelial surface layer, the glycocalyx [10].

A new perspective on evaluating the salt sensitivity in man evolved recently based on the observation that the glycocalyx of erythrocytes (red blood cells, RBC) could mimic the glycocalyx of vascular endothelium [12]. Just as in endothelium, RBC selectively bind sodium at the negatively charged surface. At extracellular sodium concentrations in the low physiological range, the remaining surface negativity is sufficient to create repulsive forces between the RBC and the vessel wall (endothelium). At high sodium concentrations, repulsive forces collapse. Then, RBC and endothelium come too close to each other. This may cause shedding of the glycocalyx, that is, damage of the RBC surface and the inner wall of the blood vessels (fig. 1). In this concept, the glycocalyx serves as a 'safety cushion' between cell surfaces. The larger the cushion, the better is the buffering power for sodium. The recently developed salt blood test (SBT) is based on this concept [13]. RBC, taken from an individual by venous puncture, are suspended in two solutions of different sodium concentrations and - in presence of some other constituents to maintain constant osmolality and to render RBC 'sticky' - allowed to sediment in vertical tubes. The ratio of the respective (high over low sodium) RBC sedimentation rates gives an estimate of the individual salt sensitivity (erythrocyte sodium sensitivity, ESS).
In order to evaluate the applicability of the SBT in medical facilities outside our research laboratory, we set up a test baggage (SBT-kit; fig. 2, inset) and sent it out to nephrologists on demand. Eight laboratories sent back their results, which are summarized in figure 2. For analysis, the data were split into two groups, normotensive patients (no history of hypertension) and hypertensive patients (with a history of hypertension and with antihypertensive treatment). Salt sensitivity was calculated for normotensive patients $(n=13)$ and the mean ESS value was taken as a reference. Then, the ESS values of the hypertensive patients was calculated and related to the respective mean ESS value of the normotensive cohort. Figure 2 shows these relative values. The grey horizontal bar exhibits the standard error zone $( \pm$ SEM). Columns (patients) that end in the grey bar were excluded from further analysis. It becomes obvious that out of 31 hypertensive patients, 15 individuals exert a high salt sensitivity (48\%) and 10 individuals a low one (32\%). This indicates that salt sensitivity in the hypertensive cohort - despite antihypertensive medication - is significantly higher as compared to the normotensive cohort (fig. 3a). Further analysis revealed that both systolic and diastolic blood pressures were found significantly increased in the hypertensive cohort - despite antihypertensive treatment (fig. 3b, c). Last but not the least, the body mass index (BMI) was found increased in the hypertensive cohort in relation to the normotensive one (fig. 3d). However, this data lack statistical significance probably due to the low number of study participants. 
Fig. 2. Salt sensitivity (ESS) obtained in 31 hypertensive patients. Individual ESS values are related to the mean value of 13 normotensive individuals and expressed in percent (ESS \%). Red bars protruding the grey zone (which is the $( \pm$ ) standard error of the normalized ESS values obtained in normotensive individuals) indicate high salt sensitivity. Green bars indicate low salt sensitivity. Grey bars ending inside the horizontal grey zone were not considered in further analyses.

Fig. 3. Data obtained in 13 normotensive and 15 hypertensive (red bars protruding the horizontal grey bar in fig. 2) individuals are analyzed. a Salt sensitivity related to normalized ESS of normotensive individuals are shown (ESS \%). In b-d systolic blood pressure, diastolic blood pressure and body mass index (BMI), respectively, of normotensive and hypertensive individuals are shown.
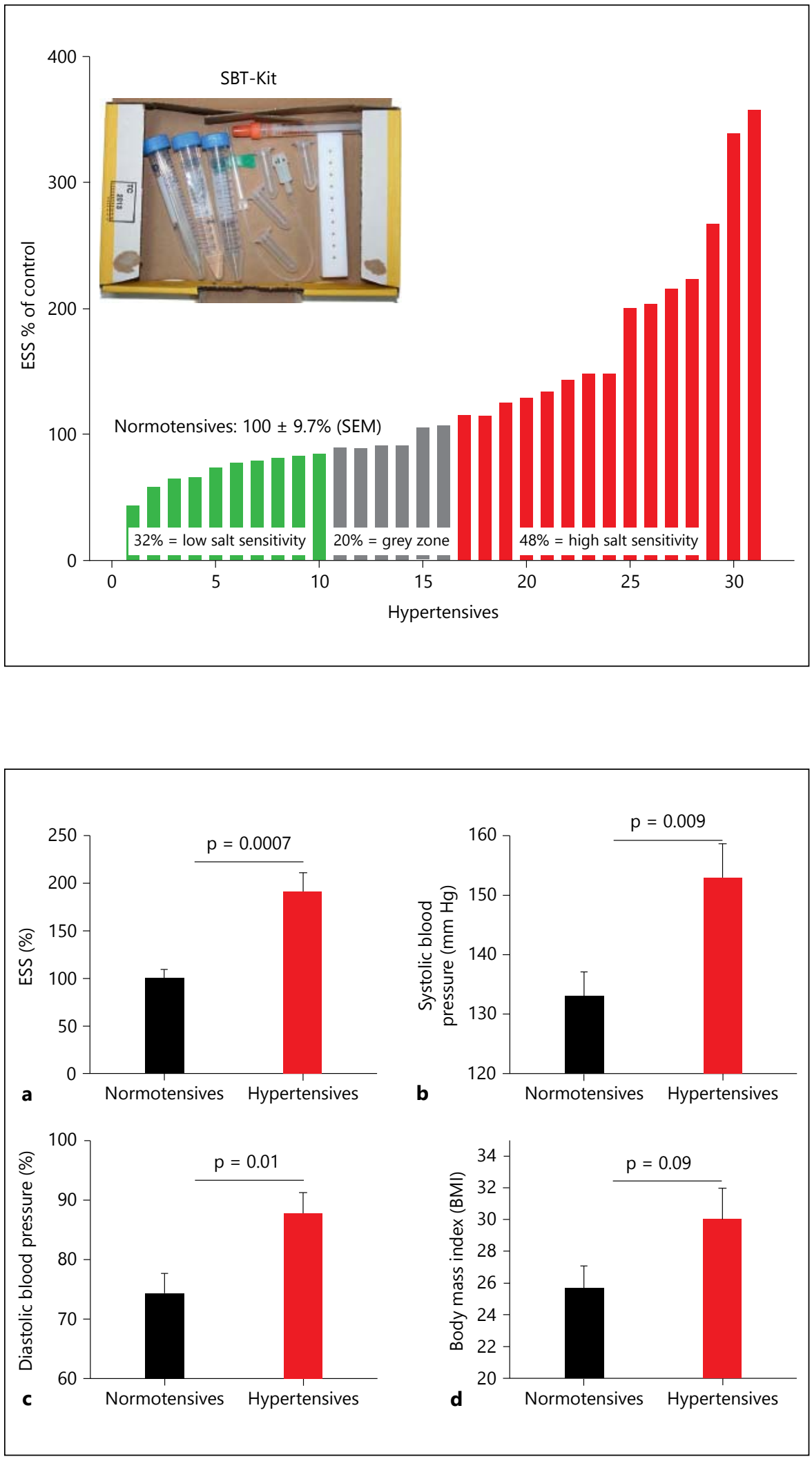


\section{Conclusion and Outlook}

The incidence of increased salt sensitivity is higher among hypertensive individuals independent of any antihypertensive therapy. However, we do not know whether the incidence of high salt sensitivity could be even higher in the absence of any antihypertensive treatment. In fact, this is likely. Although genetics could play a significant role [14], the degree of salt sensitivity of an individual is not entirely an inherited property. As shown recently in vitro [13] and in vivo [15], ESS values can be influenced. They are not constant values fixed over the whole life span of an individual. In other words, the glycocalyx of the vascular system does not slowly deteriorate with age (we could not find any dependence on either age or sex) but can also recover, at least to a certain extent. This view opens new strategies in the prevention and treatment of vasculopathies. The SBT could serve as a simple test system for follow-up studies giving feedback information to the treating physician and the treated patient. Finally, in a more direct way, the SBT could be useful as an assay system for testing the RBC surface quality of patients on dialysis. It should be remembered that the RBC glycocalyx 'reports' the quality of the inner vessel wall. Moreover, a 'poor' RBC glycocalyx (low sodium buffering power = high ESS value) could be probably 'recharged' (addition of negative charges?) during hemodialysis (unpublished observation of our laboratory). Such ' $\mathrm{RBC}$ recharge protocols' - though not yet developed - could then be tested.

\section{Acknowledgments}

We thank the following colleagues for providing data obtained with the SBT-kit: Prof. W. Grotz, Essen; Dr. C. Freudenhammer, Remagen; Dr. N. Braun, Waren; Dr. U. Lammers, Oldenburg; Dr. W. Sehnert, Dortmund; Dr. D.N. Müller, Berlin; Dr. Dr. A. Nabokow, Hannover-Münden; cand. med. O. Martin, Düsseldorf. Work was supported by Deutsche Forschungsgemeinschaft (Koselleck-grant OB63/18) and Cells-in-Motion, Cluster of Excellence (EXC 1003-CIM). We acknowledge the networking activities of the COST action TD1002. We thank Ms. Barbara Windoffer and Mr. Sergei Handel for excellent laboratory work.

\section{Disclosure Statement}

The authors declare that they have no conflict of interest. The experimental protocol was approved by the local Ethics Committee (Ärztekammer Westfalen-Lippe, approval number 2012-029f-S).

\section{References}

1 Luft FC: Sodium shows no mercy on the nanomechanics of endothelial cells. Hypertension 2014;64:231-232.

2 Weinberger MH: Salt sensitivity of blood pressure in humans. Hypertension 1996;27: 481-490.

$\checkmark 3$ Meneton P, Jeunemaitre X, de Wardener HE, MacGregor GA: Links between dietary salt intake, renal salt handling, blood pressure, and cardiovascular diseases. Physiol Rev 2005;85: 679-715.

4 Titze J, Ritz E: Salt and its effect on blood pressure and target organ damage: new pieces in an old puzzle. J Nephrol 2009;22:177-189.

$\checkmark 5$ Sanders PW: Vascular consequences of dietary salt intake. Am J Physiol Renal Physiol 2009;297:F237-F243.
6 Kleinewietfeld M, Manzel A, Titze J, Kvakan H, Yosef N, Linker RA, Muller DN, Hafler DA: Sodium chloride drives autoimmune disease by the induction of pathogenic TH17 cells. Nature 2013;496:518-522.

7 Titze J, Lang R, Ilies C, Schwind KH, Kirsch KA, Dietsch P, Luft FC, Hilgers KF: Osmotically inactive skin $\mathrm{Na}+$ storage in rats. Am J Physiol Renal Physiol 2003;285:F1108-F1117.

8 Oberleithner H, Riethmuller C, Schillers $\mathrm{H}$, MacGregor GA, de Wardener HE, Hausberg M: Plasma sodium stiffens vascular endothelium and reduces nitric oxide release. Proc Natl Acad Sci U S A 2007;104:16281-16286.

${ }_{9}$ Kusche-Vihrog K, Jeggle P, Oberleithner H: The role of $\mathrm{ENaC}$ in vascular endothelium. Pflugers Arch 2014;466:851-859.

10 Oberleithner H, Kusche-Vihrog K, Schillers $\mathrm{H}$ : Endothelial cells as vascular salt sensors. Kidney Int 2010;77:490-494.
11 Warnock DG, Kusche-Vihrog K, Tarjus A, Sheng S, Oberleithner H, Kleyman TR, Jaisser F: Blood pressure and amiloride-sensitive sodium channels in vascular and renal cells. Nat Rev Nephrol 2014;10:146-157.

12 Oberleithner H: Vascular endothelium leaves fingerprints on the surface of erythrocytes. Pflugers Arch 2013;465:1451-1458.

13 Oberleithner H, Wilhelmi M: Determination of erythrocyte sodium sensitivity in man. Pflugers Arch 2013;465:1459-1466.

14 Luft FC: Geneticism of essential hypertension. Hypertension 2004;43:1155-1159.

15 Oberleithner H: Sodium selective erythrocyte glycocalyx and salt sensitivity in man. Pflugers Arch 2014, DOI: 10.1007/s00424014-1577-0. 